MacKinnon, J. R., \& Amsel, A. Magnitude of the frustration effect as a function of confinement and detention in the frustrating situation. Journal of Experimental Psychology, 1964, 67, 468-474.

Platt, J. R., \& Senkowski, P. C. Effects of discrete-trials reinforcement frequency and changes in reinforcement frequency on preceding and subsequent fixed-ratio performance. Journal of Experimental Psychology, 1970, 85, 95-104.

Scobie, S. R., \& Fallon, D. Aftereffects of reinforcement and its omission: A fresh look at time between trials. Paper presented at the meetings of the Psychonomic Society, St. Louis, November 1972 .

Scull, J. W. The Amsel frustration effect: Interpretations and research. Psychological Bulletin, 1973, 79, 352-361.

Staddon, J. E. R. Temporal effects of reinforcement: A negative frustration effect. Learning \& Motivation, 1970, 1, 227-243.

Staddon, J. E. R., \& Innis, N. K. Reinforcement omission on fixed-interval schedules. Journal of the Experimental Analysis of Behavior, 1969,12,689-700.

Vandament, W. E., Burright, R. G., Fessenden, R. R., \& Braker, W. H. Tables of event sequences for sequential analyses of data in psychological experiments containing two-class events. Behavior Research Methods \& Instrumentation, 1970, 2, 290-296.

(Received for publication March 2, 1974.)

\title{
Reward vs extinction in discrimination reversal learning
}

\author{
BEN A. WILLIAMS \\ University of California, San Diego, La Jolla, California 92037
}

\begin{abstract}
Pigeons trained on a series of color-discrimination reversals were presented either reward or extinction single-stimulus pretraining at various points during reversal learning improvement. Extinction of the previous $\mathrm{S}+$ facilitated reversal learning more than reward of the previous $\mathrm{S}-$, with this relation remaining unchanged throughout training.
\end{abstract}

A number of experiments have addressed the question of whether simultaneous discrimination is controlled more by excitatory or by inhibitory processes. Unfortunately, no clear answer to this question is available. Several experiments have shown learning about the positive stimulus (S+) to be more effective (Ettlinger, 1960; Gardner \& Coate, 1965; Vaughter, Tyer, \& Halcomb, 1966; Hearst, 1971), while several others (Harlow \& Hicks, 1957; Stevens \& Fechter, 1968; Mandler, 1970) have favored learning about the negative stimulus (S-). These discrepant results are difficult to interpret, however, because of the variety of procedures and species used in the various experiments. Replacing the S+ or S- in transfer tests (Stevens \& Fechter, 1968; Mandler, 1968; Warren \& Kimball, 1959), the use of multiple Sts and S-s (Gardner \& Coate, 1965; Mandler, 1970), and reward vs extinction single-stimulus pretraining are a few of the procedures previously used. Similarly, the Ss have included rats, pigeons, and monkeys. Even with the same species and procedure, however, discrepant results have still been obtained (e.g., Mandler, 1970, vs Gardner \& Coate, 1965).

A notable candidate for clarifying the results is the effects of prior training. Although there are exceptions, experiments assessing excitatory/inhibitory effects during the acquisition of the initial discrimination have usually found excitatory processes to be more important, whereas those assessing such effects during reversal learning or after several discriminations have favored inhibitory processes. In addition, two experiments with primate Ss (Behar, 1961; Cross \& Brown, 1965) have shown the effects of nonreward to become greater with continued training on learning-to-learn procedures. It is unclear, however, whether these effects reflect inhibitory processes or whether they reflect the increased utilization of the cue values of response consequences.

The present experiment evaluates whether the relative roles of excitation and inhibition change for nonprimate Ss during extended training on a serial reversal problem. 


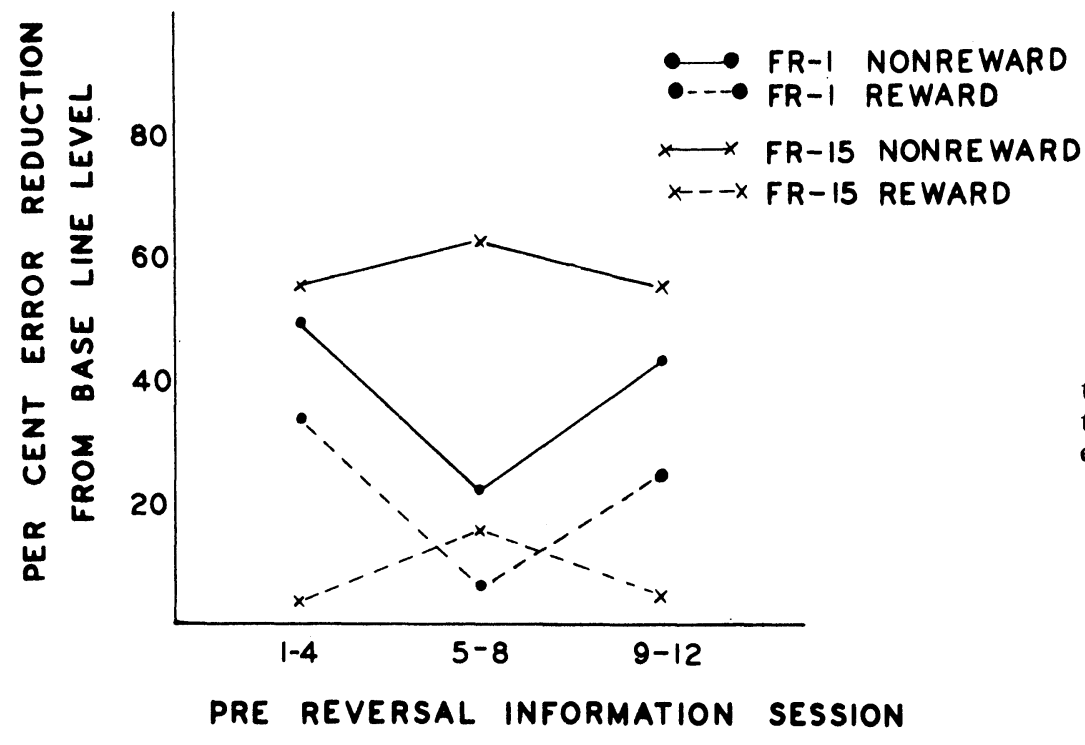

Fig. 1. Percentage decrease in errors due to pretraining. Reward and extinction pretraining effects are presented separately for each FR group.

Pigeons were presented occasional single-stimulus pretraining sessions in which either the previous $\mathrm{S}+$ was extinguished or the previous $\mathrm{S}$ - was rewarded. Any change in the effects of nonreward as a function of extended training should thus be reflected in the relative effectiveness of the two pretraining procedures.

\section{METHOD}

\section{Subjects}

Twelve White Carneaux pigeons were maintained at $80 \%$ of their free-feeding body weights. Six Ss were experimentally naive; the six others had had varying degrees of discrimination experience.

\footnotetext{
Apparatus

The apparatus was a standard operant conditioning chamber converted from an aluminum picnic cooler. On the front panel, two Gerbrands pigeon keys were mounted $10 \mathrm{~cm}$ apart, and each key required a force of at least $.15 \mathrm{~N}$ for operation. Behind each key were two 7.5-W Christmas tree light bulbs, one of which was illum inated on each trial.
}

\section{Procedure}

The Ss were initially trained for 10 sessions with a red-yellow discrimination problem. Yellow was the correct stimulus on Day 1, red was correct on Day 2, yellow on Day 3, etc. Within a daily problem, each of the two colors was present on each trial. Responses to the correct color resulted in a 2.8-sec access to the food magazine, followed by $1.2 \mathrm{sec}$ of complete darkness before the next trial. Responses to the incorrect color resulted in a blackout for the entire 4.0 -sec ITI. The positions of the colors changed randomly after a correct trial, but did not change after an incorrect trial. Training within a session continued until the bird had reached the criterion of 20 consecutively correct trials.

During red-yellow training, six Ss were trained using one peck per trial (FR 1); the six others were required to emit at least 15 pecks per trial (FR 15). With the latter procedure, correct and incorrect pecks were counted separately, and whichever first reached the FR 15 requirement determined whether the trial was correct or incorrect.

Blue and green were substituted for red and yellow, with no other changes in the daily procedure. The $12 \mathrm{Ss}$ were divided into three groups of 4 each (2 FR 1 and 2 FR 15). Two of the groups received different orders of the two pretraining conditions, while the third group received no pretraining but remained in their cages on sessions during which pretraining was scheduled.

Reward pretraining consisted of sessions in which the previous day's incorrect color was presented on one key, with the position of the color randomly determined and with the other key inoperable and darkened. When the bird pecked the color the prescribed number of times ( 1 or 15), food was delivered, followed by a 1.2-sec blackout before the next trial. Training continued until 20 reinforcements were obtained, 10 on each key. Extinction pretraining consisted of presenting the previous day's correct color on one key, again with the color's position randomly changing, but with the completion of a trial resulting only in a 4.0 -sec blackout, never in reinforcement. Again, training was continued until 20 trials were completed. Both experimental groups received both pretraining conditions in a semirandom alternation sequence. Between pretraining sessions were interspersed five reversal sessions, so that pretraining sessions occurred every 6 th day. The measure of pretraining effects was the errors to criterion occurring on the reversal session presented on the following day. Training on this schedule continued for 70 sessions.

\section{RESULTS}

Averaged over all reversals, the mean number of errors for sessions not following pretraining was 15.3 , the mean errors following extinction pretraining was 8.1 , and the mean errors following reward pretraining was 13.9. Fewer errors occurred after extinction pretraining than after reward pretraining for all eight experimental Ss (binomial probability $<.01$ ).

As a control for the effects of the different temporal intervals between reversals after pretraining and those not after pretraining (reversals after pretraining occurred $48 \mathrm{~h}$ after the previous reversal, those not after pretraining occurred $24 \mathrm{~h}$ after the preceding reversal), the errors per reversal for the four Ss which received no pretraining were computed as a function of whether the interreversal interval was 24 or $48 \mathrm{~h}$. Three of the four Ss showed slightly more errors after the 48-h interreversal interval, and the fourth showed no 
Table 1

Mean Errors Per Reversal Over the Course of Reversal Learning Improvement (Data for Separate Thirds of Training)

\begin{tabular}{ccc}
\hline Reversals & FR 1 & FR 15 \\
\hline $1-23$ & 21.8 & 19.9 \\
$24-47$ & 19.2 & 13.9 \\
$48-70$ & 12.0 & 9.3 \\
\hline
\end{tabular}

difference. The average number of errors after the 24-h interval was 11.7 , and after the 48 -h interval, 12.7 , but this difference was not statistically significant.

The question of whether the effectiveness of the two types of pretraining varied with extended training is best answered by a comparison of the relative decrease in errors due to the pretraining conditions at various points during reversal learning improvement. Since pretraining sessions occurred only every 6 th day, by averaging errors for the two reversals on each side of pretraining, it is possible to provide a baseline from which the percentage decrease in errors due to pretraining can be calculated. Shown in Fig. 1 are the averaged results of this calculation: 1.0 - (baseline error level - errors after pretraining/baseline error level) for each pretraining condition. The percentage reduction is greater for extinction pretraining for both the FR 1 and FR 15 conditions, but no meaningful trend is evident as a function of the number of reversals. The magnitude of the difference between extinction and reward pretraining does seem to vary, however, with the FR requirement. To assess the validity of these observations, the data were subjected to an analysis of variance (FR by Pretraining by Blocks). Only the pretraining factor was significant $[F(1,6)=13.93, p<.01]$. The interaction between pretraining and $F R$ was not significant $[F(1,6)=3.72, p>.05]$, although the $F$ value is of low probability.

Since the major issue addressed by this experiment was whether reward and extinction effects changed with reversal learning improvement, it is noteworthy to examine the extent of improvement during the period from which the data shown in Fig. 1 were taken. In contrast to the relative constancy of the pretraining effects, the number of errors per reversal was significantly reduced over the course of training for all eight Ss. Shown in Table 1 are the means of the two FR groups for each third of training. Errors were reduced by $40 \%-60 \%$ over the course of training from which the pretraining effects were measured.

\section{DISCUSSION}

The present results offer little support for the hypothesis that inhibitory effects of nonreward increase as a function of training on learning-to-learn procedures. Instead, the consistently greater effectiveness of extinction pretraining over reward pretraining was approximately constant at all stages of reversal learning improvement. Thus, a previous report of nonreward's increasing effectiveness (Cross \& Brown, 1965) may be limited to the procedure and/or species used in that experiment.

In addition, the present results stand in contrast to those of the only other experiment using a single-stimulus pretraining procedure with pigeons as Ss. Hearst (1971) found reward pretraining to be more effective than extinction, but his pretraining conditions were presented prior to the initial discrimination. The first pretraining experience in the present experiment occurred after several reversals had already been learned, and it is of course possible that results more similar to Hearst's would have been obtained if pretraining had been presented earlier. It seems reasonable to suppose, however, that any change in the relative effectiveness of the two pretraining conditions as a function of discrimination experience should be a graded effect, and hence detectable by the present procedure. In any event, a number of other differences between Hearst's procedure and the present experiment (e.g., differences in reinforcement schedule) might also account for the different results.

A final aspect of the present results is their relevance to the "differential forgetting hypothesis" put forth by Woodard, Schoel, and Bitterman (1971) as a general account of reversal learning improvement. Using a successive discrimination procedure, they noted that responding (at the start of a session) to the previously negative stimulus increased over successive reversals, suggesting that its inhibitory effects had been forgotten during the interreversal interval, but that a corresponding decrease in responding to the previously positive stimulus did not occur. They interpreted their results to show that inhibition was highly susceptible to proactive interference but excitation was not, and that reversal learning improvement was largely due to the former. The present experiment contrasts sharply with their findings, as the effects of reward and extinction pretraining did not change as a function of reversal learning improvement, in spite of a 24-h interval being interspersed between pretraining and the next reversal. These differences question whether changes in successive discrimination behavior are sufficiently general to be extrapolated to simultaneous discrimination procedures.

\section{REFERENCES}

Behar, I. Learned avoidance of nonreward. Psychological Reports, 1961, 9, 43-52.

Cross, H. A., \& Brown, L. T. Discrimination reversal learning in squirrel monkeys as a function of number of acquisition trials and prereversal experience. Journal of Comparative \& Physiological Psychology, 1965, 59, 429-431.

Ettlinger, G. Discrimination learning theory: Excitatory versus inhibitory tendencies in monkeys. Quarterly Journal of Experimental Psychology, 1960, 12, 41-44.

Gardner, R. A., \& Coate, W. B. Reward vs. nonreward in a simultaneous discrimination. Journal of Experimental Psychology, 1965, 69, 579-582.

Harlow, H. F., \& Hicks, L. H. Discrimination learning theory: Uniprocess vs. duoprocess. Psychological Review, 1957, 64, 107-109.

Hearst, E. Differential transfer of excitatory versus inhibitory pretraining to intradimensional discrimination learning in pigeons. Journal of Comparative \& Physiological Psychology, pigeons. Journal of

Mandler, J. M. Overtraining and the use of positive and negative stimuli in reversal and transfer. Journal of Comparative \& Physiological Psychology, 1968, 66, 110-115.

Mandler, J. Two choice discrimination learning using multiple stimuli. Learning \& Motivation, 1970, 1, 261-266.

Stevens, D. A., \& Fechter, L. D. Relative strengths of approach and avoidance tendencies in discrimination learning of rats trained under two types of reinforcement. Journal of Experimental Psychology, 1968, 76, 489-491.

Vaughter, R. M., Tyer, Z., \& Halcomb, C. Discrimination performance in deprived and non-deprived rats as a function of pre-discrimination information. Psychonomic Science, 1966, 5, 199-200.

Warren, J. M. \& Kimball, H. Transfer relations in discrimination learning by cats. Journal of Comparative \& Physiological Psychology, 1959, 52, 336-338.

Woodard, W. T., Schoel, W., \& Bitterman, M. E. Reversal learning with singly-presented stimuli in pigeons and goldfish. Journal of Comparative \& Phy siological Psychology, 1971, 76, 460-467.

(Received for publication March 21, 1974.) 